\title{
An improved Shuffled frog leaping Algorithm for Time-table Problems
}

\author{
Hujun Meng1,a Yueguang Li 2,b \\ 1Gansu Normal University for Nationalities, Hezuo, gansu, 747000, \\ China \\ 2Gansu Normal University for Nationalities, Hezuo, gansu, 747000, \\ China \\ aYueguangli7@163.com,b Yueguangli7@sohu.com
}

\begin{abstract}
This paper proposes a shuffled frog leaping algorithm based on population diversity feedback. The algorithm is used to solve the Time-table Problem, the results show that the improved shuffled frog leaping algorithm has reliable convergence, high convergence rate and solution precision.
\end{abstract}

Keywords: Time-table Problem; Improved Shuffled frog leaping algorithm; Diversity; Intelligent algorithm

\section{Introduction}

Shuffled frog leaping algorithm is a simulation of the frog group foraging behavior of modern heuristic intelligent algorithm. The algorithm has flexible structure frame, strong global information sharing and interaction. Muzaffar M. Eusuff and Kevin E. Lansey supposed the algorithm in 2000 and it is used to solve water resource in network distribution problem ${ }^{[1]}$. Academic conducted extensive research on its theory and application. At present on the results of theoretical research of Shuffled frog leaping algorithm is not much and related research: Elbeltagi Emad compared the searching mechanism of the genetic algorithm and shuffled frog leaping algorithm in 2005, the experimental results show that the shuffled frog leaping algorithm in solving some problems of 
continuous functions have better performance than genetic algorithm ${ }^{[2]}$. Eusuff studied the system theory of basic shuffled frog leaping algorithm in 2006, the perfect shuffled frog leaping algorithm was used to solve typical combinatorial optimization problems ${ }^{[3]}$. Elbeltagi Emad proposed an improved shuffled frog leaping algorithm in 2007, proposed the parameter of accelerate search scope, analyzed the positive role of the new parameters, and solved discrete optimization problem and continuous optimization problem ${ }^{[4]}$. In 2008, Zhang introduced the individual cognition learning ability of PSO algorithm into shuffled frog leaping algorithm, thereby improving the individual learning ability of shuffled frog leaping algorithm ${ }^{[5]}$. In 2009, Xuan Zongyi due to the discrete optimization problem, built the behavior describe of knapsack problem based on $0 / 1$ code, and the improved Shuffled frog leaping algorithm was used to solve $0-1$ knapsack problem ${ }^{[6]}$.

Time-table Problem of Universities is a many factor of the global optimization problem, it is a NP- complete problem ${ }^{[7]}$. Since Time-table Problem of Universities involved in many information, the methods for Time-table Problem of Universities are the classical algorithm, structure algorithms and intelligent optimization algorithms. Among them, the classical algorithms, such as branch and bound method, dynamic programming method the exact solutions of problems can be obtained, but the classical algorithms are limited by the problem size and computing complexity, only suitable for solving small problems. Constructive algorithms, such as NEH method, Rajendran method, the methods can set up the problem of scheduling solution quickly, but the structure complex, and usually the solution quality is poor. Intelligent optimization algorithms, such as genetic algorithm ${ }^{[8]}$, ant colony algorithm ${ }^{[9]}$, particle swarm optimization algorithm ${ }^{[10]}$, immune algorithm ${ }^{[11]}$ and a variety of hybrid algorithm etc $^{[12]}$. The intelligent algorithms have been paid more and more attention, they are used to solve combinatorial optimization and NP. In order to solve the above problems, this paper puts forward an improved Shuffled frog leaping algorithm to solve Time-table Problem of Universities.

\section{Shuffled frog leaping algorithm based on diversity feedback (SFLADF)}




\subsection{Attract operation and exclusive operation}

How to measure and dynamic regulate population diversity and its effect in the mixed iteration is the main question of shuffled frog leaping algorithm need to solve. In view of this, this paper presents the shuffled frog leaping algorithm based on diversity feedback (SFLADF) schematic diagram as shown in Figure 1. In the schematic diagram of this algorithm, the frog population is a typical closed-loop automatic control system. The diversity controller uses effectively diversity evaluation index to calculate the diversity of the population in the system and uses the feedback mechanism to determine increase population diversity or reduce the population diversity according to the real-time population diversity, and so as to control the output strategies of group optimizer, dynamic adjust the frog individual behavior, improve the population diversity through adjusting the frog's behavior and promote a higher quality solutions.

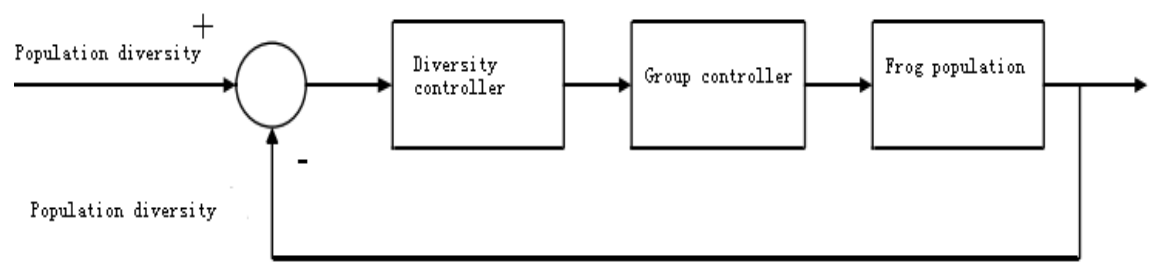

Fig.1 The diagram of diversity feedback for S FLA

In the shuffled frog leaping algorithm based on diversity feedback, diversity controller adjusts the population diversity through population dynamic optimizer. The SFLADF of this paper defines the two states of frog population: attract state and excluded state, also defines the upper limit Dhigh of the diversity and lower limit Dlow of the diversity; When the diversity of population is higher than Dhigh, the frog population begins to population center for attracting operation; when the diversity of population is lower than Dlow, the frog population begins to stay away from population centers for exclusive operation, make the frog individual divergence to other locations. Effectively overcome the basic SFLA easily fall into local optimum problem. 
For the attract operation and exclusive operation of frog populations, design the following method.

(1) Attract operation

In the basic SFLA algorithm, the optimal position of individual information sharing is the key of algorithm success, if increasing the utilization rate of frog individual optimal position information, the performance and searching ability of the algorithm will improve ${ }^{[41]}$. Through referencing to the "elite group" thought of genetic algorithm, put forward the attraction state control method of the population diversity. That is, the frogs of optimal position will replace $K(k<P)$ frogs of the worst fitness, prompt the frogs quickly closes to the optimal location.

If

$x_{i}=\left(x_{i 1}, x_{i 2}, \ldots, x_{i D}\right) \quad$ satisfies $\quad f\left(x_{1}\right)>f\left(x_{2}\right)>\ldots>f\left(x_{p}\right)$, then $\Omega_{k}=\left(x_{1}, x_{2}, \ldots, x_{k}\right), k<p$ ( $p$ is the size of the group), $f_{x}$ for the individual fitness, fitness is in descending order.

(2) Exclusive operation

When the diversity of population is less than Dlow, the frogs of the worst position take the Gauss variation perturbation operation to produce new position, then the update strategy:

$$
\begin{aligned}
& \text { If } \quad \text { Diversity }(\mathrm{t})<\text { Dlow } \\
& D(t+1)=(1+\text { gauss }(0,1)) \times \text { rand } \times\left(X_{b}-X_{w}\right) \\
& X_{w}(t+1)=X_{w}(t)+D(t+1) \\
& D(t+1)=- \text { rand } \times\left(X_{g}-X_{w}\right)
\end{aligned}
$$

Among them, Gauss $(0,1)$ for the Gauss distribution function of $u=0, \sigma=1$. 


\subsection{The realization of algorithm}

The algorithm steps described as follows:

Parameter setting: the size of population is $p$, and the random initialization, population size is $m$, each group contains $n$ individuals, the number of iterations in family is $G_{n}$ and mixed iteration $G_{m}$;

For $\mathrm{i}=1: \mathrm{Gm}$

Calculate the population diversity $\operatorname{Diversity}(i)$

If (Diversity (i) > Dhigh)

The implementation of the attract operation of algorithm;

If (Diversity $($ i $)<$ Dlow)

The implementation of the exclusive operation of algorithm, use the strategy of "divergence" behavior;

Else

Search with the basic SFLA

End

End

\section{The simulation experiment and analysis}

In order to validate the effectively and solving the ability of the algorithm, this paper selects Gansu Normal College for Nationalities (4grades,240 classes, 700 teachers) as an example to test.

Through extensive simulations, the parameters of this algorithm as following: population size $P=200$, the number of frog $m=20$ within population groups, $n=10, G_{n}=10, G_{m}=500$, the dimension of test function is 30 , the upper limit of Dhigh is 0.20, the lower limit of Dlow is 0.001. Figure 2 is the population diversity of function change curve. Among them, the vertical axis represents the value of population diversity of function, the abscissa values for the evolution algebra. 


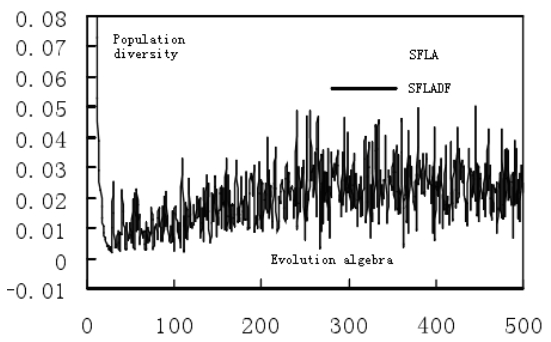

Fig. 2 Population diversity

Seen from the data in Figure 2, Basic shuffled frog leaping algorithm easily trapped into local optimal solution. Improved shuffled frog leaping algorithm is proposed in this paper, compared with the basic shuffled frog leaping algorithm, it has good ability to maintain the population diversity, and with the increase of evolutionary algebra, it searches the ability of the optimal solution is in the unceasing enhancement. It shows the diversity of the improved algorithm is effective.

\section{Conclusions}

This paper proposes a shuffled frog leaping algorithm based on population diversity feedback, the algorithm is applied to solve the Time-table Problem, the performance of the algorithm has been improved obviously. Experiments show that the diversity of the improved algorithm is effective.

\section{References}

[1] Muzaffar M. Eusuffand Kevin E. Lansey. Optimization of water distribution net work design using the shuffled frog leaping algorithm [J].Journal of Water Resources Planning and Management. 2003, 129(3): 210-225.

[2] Ebeltagi E, Hegazy T, Grierson D. Comparison among five evolutionary based optimization algorithm [J]. Advanced Engineering Informatics, 20 05, 19 (1):43-53.

[3] Eusuff MM, Lansey KE, Pasha F. shuffled frog leaping algorithm: a memetic meta-heuristic for discrete Optimization. Engineering Optimization, 2006, 38(2):129-154 . 
[4] Elbeltagi E, Hegazy T, Grierson D. A modified shuffled frog leaping algorithm: Applications to project management [J]. Structure and Infra structure Engineering, 2007, 3(1):125-130.

[5] Zhang Xuncai, Hu Xuemei, Cui Guangzhao. An improved shuffled frog leaping algorithm with cognitive behavior[C].Proceedings of the 7th World Congress on Intelligent Control and Automation. Chongqing, 2008: 6197-6202.

[6] Xuan Zongyi, Zhang Cuijun. The knapsack problem solving based on shuffled frog leaping algorithm [J]. Science technology and engineering, 2009, 9(15): 4363- 4365.

[7] Garey M R, Johnson D S. Computers and Intractability: A Guide to the Theory of NP-Completeness[M]. San Franciso,CA: Freeman,4,109-122(1979).

[8] Holland J H. Genetic Algorithms and the Optimal Allocation of Trials [J]. SIA M J Computer, 1973: 2 (2): 89-104.

[9] Colorni A, Dorigo M, Maniezzo V. Distributed Optimization by Ant Colonies[A] . Proc 1st European Conf Artificial Life Plans[C]. France: Elsevier, 1991: 134-142.

[10] Eberhart R, Kennedy J. A New Optimizer Using Particles Swarm Theory[A]. Proc 6th Int Symposium on Micro Machine and Human Science[C]. Nagoya: IEEE Service Center, Piscataway, 1995: 39-43.

[11] Dasgupta D, Forrest S. Artificial Immune Systems and Their Applications [M]. Berlin: Spring-Verlag, 1998:267-277.

[12] Wang L. Intelligent Optimization Algorithms Applications [M]. Beijing: Tsinghua University Press,2001. 